\title{
Ki-67 is an independent predictor of prostate cancer death in routine needle biopsy samples: proving utility for routine assessments
}

\author{
Solène-Florence Kammerer-Jacquet ${ }^{1,2} \cdot$ Amar Ahmad $^{3} \cdot$ Henrik Møller $^{4} \cdot$ Holly Sandu $^{3} \cdot$ Peter Scardino $^{5}$. \\ Geraldine Soosay $^{6} \cdot$ Luis Beltran $^{1} \cdot$ Jack Cuzick $^{3} \cdot$ Daniel M. Berney $^{1}$
}

Received: 14 November 2018 / Revised: 6 March 2019 / Accepted: 12 March 2019 / Published online: 11 April 2019

(c) United States \& Canadian Academy of Pathology 2019

\begin{abstract}
Standard clinical parameters fail to accurately differentiate indolent from aggressive prostate cancer. Our previous studies showed that immunohistochemical testing for Ki-67 improved prediction of prostate cancer death in a previous cohort of conservatively treated clinically localized prostate cancer. However there is a need for validation of usage with whole biopsy sections rather than tissue micro-arrays for use in routine diagnostics. Prostate cancer biopsy cases were identified in the UK, between 1990 and 2003, treated conservatively. Tumor extent and prostate-specific antigen (PSA) serum measurements were available. Biopsy cases were centrally reviewed by three uropathologists and Gleason conformed to contemporary ISUP 2014 criteria. Follow-up was through cancer registries up until 2012. Deaths were divided into those from prostate cancer and those from other causes. The percentage of Ki-67 in tumor cells was evaluated by immunohistochemistry on whole biopsy sections and was available for 756 patients. This percentage was used in analysis of cancer specific survival using a Cox proportional hazards model. In univariate analysis, the interquartile hazard ratio (HR) (95\% confidence intervals) for continuous $\mathrm{Ki}-67$ was $1.68(1.49,1.89), \chi_{1}^{2}=47.975, P<0.001$. In grade groups 1 and 2 , continuous $\mathrm{Ki}-67$ was a statistically significant predictor of time to death from prostate cancer, HR $(95 \% \mathrm{CI})=1.97(1.34,2.88), \chi_{1}^{2}=9.017, p=$ 0.003. In multivariate analysis, continuous $\mathrm{Ki}-67$ added significant predictive information to that provided by grade groups, extent of disease and serum PSA, HR $(95 \% \mathrm{CI})=1.34(1.16,1.54), \Delta \chi_{1}{ }^{2}=13.703, P<0.001$. We now advocate the introduction of $\mathrm{Ki}-67$ as a viable and practicable prognostic biomarker in clinical practice. The association of Ki-67 with mortality was highest in grade groups 1 and 2, showing that Ki-67 can be used as a routine biomarker in patients being considered for active surveillance.
\end{abstract}

Supplementary information The online version of this article (https:// doi.org/10.1038/s41379-019-0268-y) contains supplementary material, which is available to authorized users.

Solène-Florence Kammerer-Jacquet

jacquet.sf@gmail.com

1 Department of Molecular Oncology, Barts Cancer Institute, Queen Mary University of London, London EC1A 7BE, UK

2 Department of Pathology, University Hospital of Rennes, Université de Rennes 1, Université Bretagne Loire, 35000 Rennes, France

3 UK Centre for Cancer Prevention, Wolfson Institute of Preventive

\section{Introduction}

Prostate cancer remains the most common non-cutaneous malignancy in men. However many patients do not require radical treatment and will die from unrelated causes. Consequently a significant proportion of patients undergo radical treatment without need $[1,2]$. Conversely, some patients will fail treatments notwithstanding the use of

Medicine, Queen Mary University of London, London EC1A 7BE, UK

4 Cancer Epidemiology and Population Health, King's College London, London SE1 9RT, UK

5 Department of Urology, Memorial Sloan-Kettering Cancer Center, New York, NY 10065 NY, USA

6 Department of Pathology, Queen's Hospital, RM7 0AG Romford, Essex, UK 
combined modality approaches [3]. Therefore decision making in early prostate cancer remains difficult even using current the clinical parameters of serum prostate-specific antigen (PSA), routine histopathology, imaging and patient age and performance status.

In spite of extensive research, few molecular markers have been translated into routine use in genito-urinary oncology. Any new biomarker must give further predictive ability beyond that available by using current known, easily measured parameters, and the data must differentiate patients into groups for which different options can be selected from active surveillance to radical treatment.

The most promising immunohistochemical biomarker identified for prostate cancer is $\mathrm{Ki}-67$, a cell proliferation marker, expressed in G1, S, G2 and M phases of the cell cycle being absent in resting cells (G0). As the grading system in prostate cancer is pattern based and does not consider the proliferation rate of the cells, it is possible that measuring the cell proliferation rate in prostate tumors could yield additional prognostic information. $\mathrm{Ki}-67$ has been shown to be an independent predictor of prostate cancer outcome in a large number of studies on systematic review [4].

Our previous work, on the Trans-Atlantic Prostate Group cohort of conservatively managed prostate cancers diagnosed by transurethral resection of the prostate and needle biopsies showed that Ki-67 was an independent prognostically significant marker when PSA, Gleason score, and tumor extent were also considered [5, 6]. However, this cohort included many transurethral resection of the prostate patients, and biopsy patients were often sub-optimally sampled. Also these and other studies utilize tissue micro arrays and do not reflect the methods, which will be used in routine pathology laboratories to assess Ki-67.

Our hypothesis was that immunohistochemical assessment of Ki-67, in a conservatively treated cohort, would be of independent prognostic value with prostate cancer death at the end point, using a methodology which could be directly translated to routine pathology laboratories and thus into clinical use.

\section{Materials and methods}

\section{Patients}

Cases of prostate cancer were identified from three cancer registries in Great Britain. Within each region, collaborating hospitals were sought and cases from these hospitals were reviewed. Men were included in this study if they were under age 76 years at the date of diagnosis and had clinically localised prostate cancer diagnosed by needle biopsy between 1990 and 2003 inclusively. The median date of diagnosis was May 2002. Patients treated by radical prostatectomy or radiation therapy within 6 months of diagnosis were excluded. In addition, those with objective evidence of metastatic disease (by bone scan, X-ray, radiograph, CT scan, MRI, bone biopsy, lymph node biopsy and pelvic lymph node dissection) or clinical indications of metastatic disease (including pathologic fracture, soft-tissue metastases, spinal compression, or bone pain), or a PSA measurement over $100 \mathrm{ng} \mathrm{ml} / 1$ at or within 6 months of diagnosis were also excluded. Men who had hormone therapy before the diagnostic biopsy were also excluded, because of the influence of hormone treatment on Gleason pattern. We also excluded men who died within 6 months of diagnosis, or had $<6$ months of follow-up. Follow-up was conducted through the cancer registries and the cut-off date was 31 December 2012. Deaths were divided into those from prostate cancer and those from other causes, according to World Health Organisation standardised criteria (WHO, 2010). National ethics approval was obtained from the Northern Multicenter Research Ethics Committee, followed by local ethics committee approval at each of the collaborating hospitals.

\section{Histology}

Original histological specimens from the diagnostic procedure were requested and centrally reviewed by a panel of three expert urological pathologists to confirm the diagnosis of adenocarcinoma and to reassign Gleason score using of a contemporary and consistent interpretation of the Gleason scoring system [7, 8] and reclassified into grade groups [8].

\section{KI-67 immunohistochemistry}

Diagnostic formalin-fixed paraffin-embedded needle biopsy tissue blocks and slides were requested. Biopsies showing the highest Gleason grade were selected. Corresponding sections were immunoassayed for Ki-67 using MIB-1 antibody, DAKO, Carpinteria, CA, USA, and attempted to reduce pre analytical variables by following recommendations used in the assessment of $\mathrm{Ki}-67$ in breast cancer by using the recommended methods and platforms [9]. Briefly, cells were scored in a semi-quantitative manner, by an expert prostatic pathologist, and the mean percentage of positive cells was estimated as the proportion of Ki-67 stained malignant cells, in a manner similar to that used in routine pathology departments for the assessment of proliferation index in other organs $[9,10]$. Corresponding $\mathrm{H} \& \mathrm{E}$ slides were simultaneously reviewed. All nuclear immunostaining was recorded as positive and was clearly either strongly positive or negative. Normal tonsil was used as a positive control. The technique was designed to be robust for any pathology laboratory with experience in immunohistochemistry and allow results to be directly comparable to our previous work. 
Table 1 Distribution of variables in the needle biopsy cohort

\begin{tabular}{lc}
\hline Variable & Median (IQR) or n(\%) \\
\hline Patient's age (years) & $70.93(66.77-73.61)$ \\
Grade group (GG) & $181(24)$ \\
GG1 & $246(33)$ \\
GG2 & $179(24)$ \\
GG3 & $49(6)$ \\
GG4 & $101(13)$ \\
GG5 & $1.57(3.44-0.85)$ \\
PSA (ng/ml per 10\%) & $5.56(8.57-3.33)$ \\
Extent of disease (per 10\%) &
\end{tabular}

\section{Statistical analysis}

Univariate and multivariate analysis was performed using a Cox proportional hazards model with the primary end point of analysis defined as death from prostate cancer. Observations stopped either on the date of death from other causes or on the date of last follow-up. Covariates included were age at diagnosis, grade groups, Ki-67 average, baseline PSA value (defined as last pre-diagnostic PSA measurement within 6 months before diagnosis), extent of disease (percentage of positive cores). Receiver operating characteristic curve analysis was performed at ten-year survival to assess the predictive cut-off value for $\mathrm{Ki}-67$ expression used to analyse Ki-67 as a binary variable. All $p$ values were two-sided with significance set at $\alpha<0.05$. Statistical analyses were performed using R (version 3.4.3, The $\mathrm{R}$ Foundation for Statistical Computing, Vienna, Austria).

\section{Results}

\section{Cohort characterization}

A total of 756 patients were available for analysis. Out of them, 294 patients received no initial therapy, while 462 received early hormonal therapy only as an initial treatment. The mean, median and interquartile range of patient age, grade groups, serum PSA and extent of disease is shown in Table 1. Follow-up for prostate cancer death was until 31 December 2012 with 145 (20\%) patients who died from their cancer. The median follow-up time was 9.6 years (IQR: 11.2-6.6).

\section{Ki-67 expression}

The mean percentage of Ki-67 was 5\%, $53(7 \%)$ scored as less than $1 \%, 429(57 \%)$ as between 2 and $5 \%, 175(23 \%)$ as

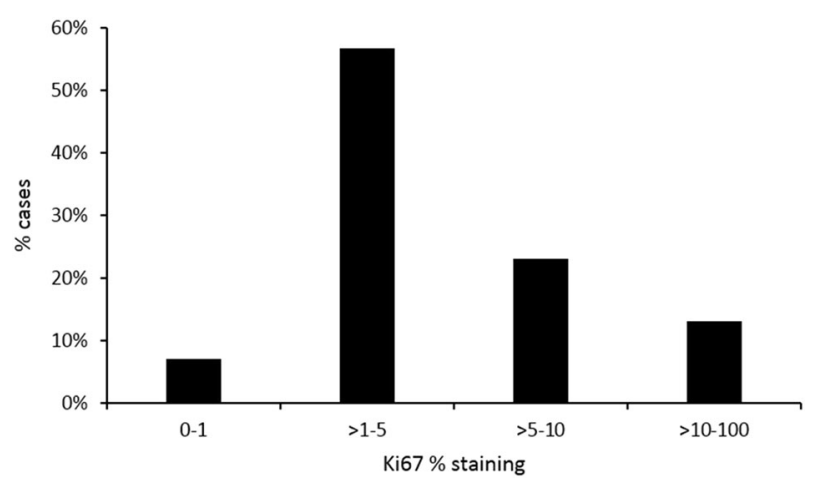

Fig. 1 Distribution of Ki-67\% staining in cases, $n=756$

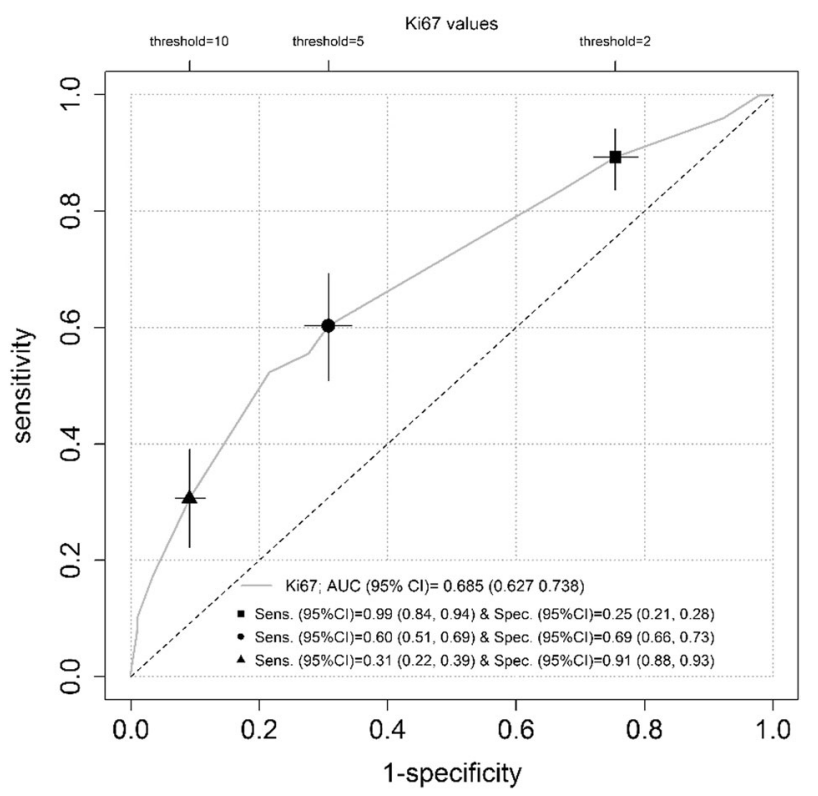

Fig. 2 Survival Receiver Operating Characteristics curve at 10 years. The sensitivity $(95 \% \mathrm{CI})$ and specificity $(95 \% \mathrm{CI})$ were reported for a cut-off value of 2 (black square), 5 (black circle) and 10 (black triangle) of the Ki-67. All 95\% confidence intervals (CI) were based on 1000 bootstrap replicates

between 6 and 10\% and 99 (13\%) as more than 10 (Fig. 1 and Supplementary Figure). The majority of deaths $n=80$ (55\%) were in the high $\mathrm{Ki}-67$ groups $(>5 \%, n=274)$. The Receiver Operating Characteristics curve analysis (AUC $=0.685,95 \%$ CI: $0.637-0.738$ ) identified $5 \%$ as the most predictive cut-off value for Ki-67 expression with a sensitivity of $60 \%$ and a specificity of $69 \%$. This cut-off was subsequently used in univariate and multivariate analysis (Fig. 2).

\section{Univariate analysis}

In univariate analysis, grade groups, PSA, extent of disease and $\mathrm{Ki}-67$ were all significant predictors of prostate cancer death (Ki-67: HR $=1.68,95 \%$ CI: $1.49-1.89)$, but not the age of patient (Table 2). Kaplan-Meier cancer specific 
Table 2 Univariate and multivariate analysis for time to death from prostate cancer in a conservatively managed needle biopsy cohort

\begin{tabular}{|c|c|c|c|c|c|c|c|}
\hline \multirow[t]{2}{*}{ Variable } & \multicolumn{4}{|l|}{ Univariate } & \multicolumn{3}{|l|}{ Multivariate } \\
\hline & $\mathrm{IQR}^{\mathrm{a}} \mathrm{HR}(95 \% \mathrm{CI})$ & $\operatorname{LR} \chi_{1}^{2}$ & $p$-value & c-index ${ }^{b}$ & $\mathrm{IQR}^{\mathrm{a}} \mathrm{HR}(95 \% \mathrm{CI})$ & $\operatorname{LR} \chi_{1}^{2 c}$ & $p$-value \\
\hline Grade group & $1.68(1.50,1.89)$ & 74.56 & $<2.2^{\mathrm{a}} 10^{-16}$ & 0.716 & $1.38(1.20,1.59)$ & 74.56 & $<2.2^{\mathrm{a}} 10^{-16}$ \\
\hline Ki-67 (continuous) & $1.68(1.49,1.89)$ & 47.97 & $4.3^{\mathrm{a}} 10^{-12}$ & 0.665 & $134(1.16,1.54)$ & 19.85 & $8.38^{\mathrm{a}} 10^{-06}$ \\
\hline Extent & $3.21(2.35,4.38)$ & 59.96 & $97^{\mathrm{a}} 10^{-15}$ & 0.690 & $1.74(1.21,2.50)$ & 14.29 & 0.0002 \\
\hline PSA & $1.66(1.43,1.93)$ & 36.90 & $1.24^{\mathrm{a}} 10^{-09}$ & 0.660 & $1.16(0.98,1.38)$ & 2.77 & 0.096 \\
\hline Age & $1.17(0.93,1.47)$ & 1.81 & 0.178 & 0.527 & & & \\
\hline LR $\chi_{4}^{2}(p$-value $)$ & & & & & $111.469(<2 \mathrm{e}-16)$ & & \\
\hline c-index ${ }^{\mathrm{b}}(95 \% \mathrm{CI})$ & & & & & $0.757(0.707,0.808)$ & & \\
\hline
\end{tabular}

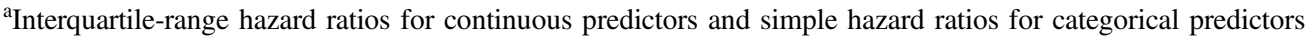

bHarrell's c-index

${ }^{\mathrm{c}}$ Terms added sequentially (first to last)

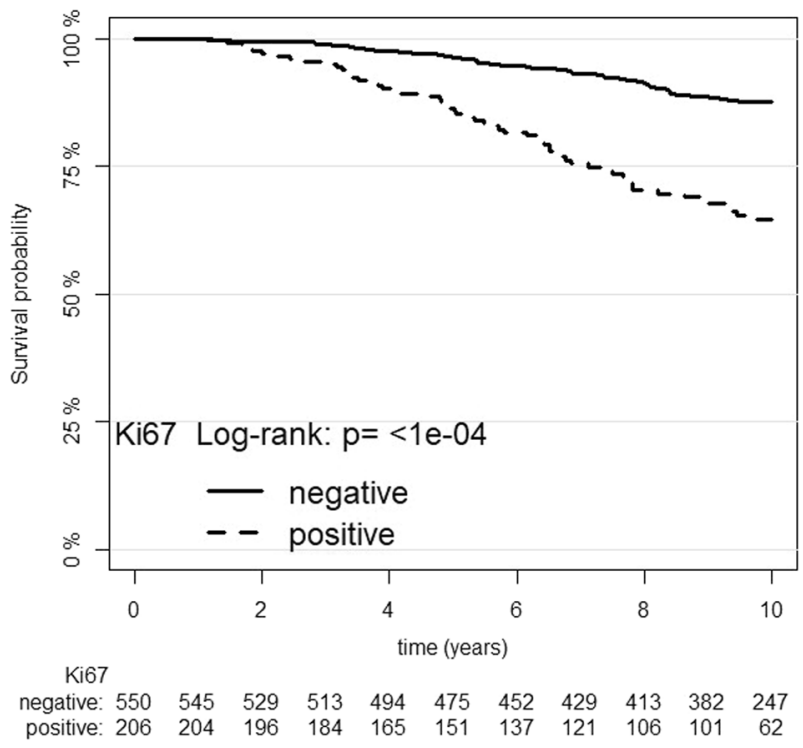

Fig. 3 Kaplan-Meier estimates of prostate cancer death according to Ki-67 (negative: $\leq 5$ and positive: $>5$ ) at 10 -year death rate from prostate cancer

survival curves for Ki-67 in two groups $(\leq 5$ or $>5 \%)$ and showed significant difference between the two groups with an $\mathrm{HR}=2.65$ (95\% CI (1.91-3.69), log-rank test $=33.46$, $\left.p=7.3 \times 10^{-09}\right)$, Fig. 3. Ki-67 was stratified by grade groups using Forest plot graphing (Fig. 4). It was found that the most predictive $\mathrm{Ki}-67$ information was in grade groups 1 and 2 compared to the other groups $(\mathrm{HR}=1.1,95 \% \mathrm{CI}$ : 1.04-1.16).

\section{Multivariate analysis}

In multivariate analysis, continuous and dichotomous Ki-67 variables added significant predictive information to that provided by grade groups and PSA alone (continuous $\mathrm{Ki}-$ $67: \mathrm{HR}=1.34,95 \%$ CI: $1.16,1.54, \mathrm{Ki}-675 \%: \mathrm{HR}=1.79$,
95\% CI: $1.27,2.53)$. The covariates extent of disease and PSA were slightly less informative (Table 2).

\section{Discussion}

The growing evidence concerning prostate cancer overdiagnosis and over-treatment emphasizes the need for accurate disease aggressiveness stratification [2]. Owing to limitations of standard clinicopathological and biological parameters, there is an unmet need for robust biomarkers that may assist clinicians in deciding therapeutic strategy.

Ki-67 is an attractive biomarker from a technical perspective due to its universal use and reproducible interpretation compared to other prostatic tissue biomarkers [11]. Indeed, it is widely used in routine practice, affordable by academic and non-academic histopathology departments, and easy to measure in a semi-quantitative manner. Immunochemistry has the advantage over other molecular techniques that it is easily applied to paraffin-embedded tissue and has a very low 'failure' rate, unlike fluorescence.

Interestingly, results of immunohistochemical analysis of a single marker of cell proliferation, Ki-67, could correlate with those of a commercially available cell-cycle progression score (Prolaris ${ }^{\circledast}$ ), a molecular test that evaluates RNA signature of several cell-cycle progression genes, as one of the cell-cycle progression genes codes for $\mathrm{Ki}-67$ [12]. If this hypothesis is validated, Ki-67 immunohistochemistry, with easier and broader worldwide implementation, well-established analytical performance, and reduced costs could help select patients for those the molecular test is relevant.

Several studies have reported Ki-67 as an independent prognostic factor for prostate cancer. Nevertheless, there is a considerable heterogeneity among studies, including different endpoints, cohort types, sample sizes and cut-offs. The studies with cancer specific survival as an endpoint are compared in Table 3 [5, 6,13-20]. 
Fig. 4 Forest plot graphing the Ki-67 hazard ratio in different grade groups

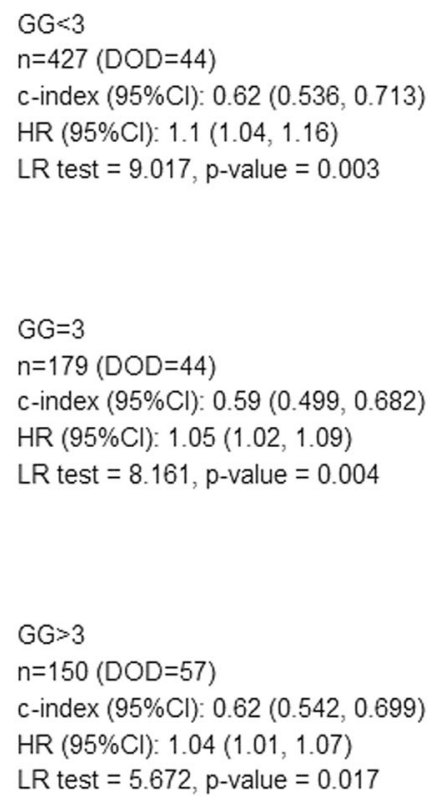

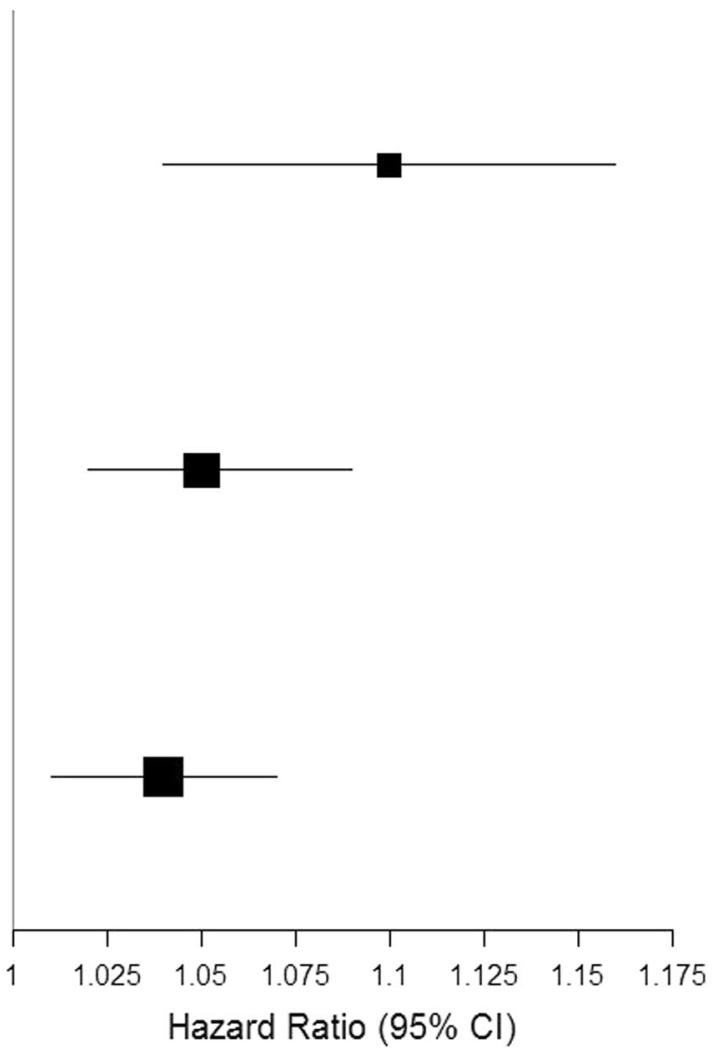

Table 3 Published reports of the prognostic value of Ki-67 in multivariate analyses with hazard ratio's (HR) after adjument for covariates with prostate cancer-survival as end point

\begin{tabular}{|c|c|c|c|c|c|c|}
\hline Reference & Cohort type & Cohort size & Cutoff $(\%)$ & p-value & $\operatorname{HR}(95 \% \mathrm{CI})$ & Covariates \\
\hline Stattin et al. [13] & TURP, WW & 125 & 3 & 0.0023 & $2.51(1.39-4.53)$ & G T A \\
\hline Li et al. [14] & $\mathrm{RX}$ & 108 & 3.5 & 0.009 & $4.24(1.43-12.57)$ & G T Tx \\
\hline Pollack et al. [15] & $\mathrm{RX}$ & 496 & cont & 0.001 & $3.64(2.15-6.16)$ & G T P Tx \\
\hline Pollack et al. [15] & $\mathrm{RX}$ & 496 & 7.1 & 0.0174 & $2.07(1.14-3.76)$ & G T P Tx \\
\hline Berney et al. [5] & TURP, WW & 685 & 5 & 0.0003 & $1.83(1.33-2.53)$ & G P Px \\
\hline Khor et al. [16] & $\mathrm{RX}$ & 637 & 11.3 & 0.0007 & $2.35(1.43-3.85)$ & G T A Tx \\
\hline Fisher et al. [6] & BX, WW & 293 & 10 & 0.011 & $2.66(1.36-5.2)$ & G P \\
\hline Verhoven et al. (2013) & $\mathrm{BX} \mathrm{RX}$ & 468 & 6.2 & 0.0064 & $2.91(1.35-6.26)$ & A G P T \\
\hline Tollefson et al. [17] & $\mathrm{BX}$ & 451 & cont & 0.001 & $1.11(1.07-1.15)$ & G Px \\
\hline Tretiakova et al. [18] & $\mathrm{RP}$ & 1004 & cont & 0.02 & $1.10(1.02-1.18)$ & G P Px \\
\hline Tretiakova et al. [18] & $\mathrm{RP}$ & 1004 & 5 & ns & & G P Px \\
\hline Lobo et al. [19] & $\mathrm{BX}$ & 189 & 5.33 & $<0,05$ & $1.91(1.01-3.57)$ & A G T \\
\hline Kammerer-Jacquet et al. (this paper) & $\mathrm{BX}, \mathrm{WW}$ & 756 & cont & 0.001 & $1.08(1.06-1.10)$ & A E G P \\
\hline Kammerer-Jacquet et al (this paper) & $\mathrm{BX}, \mathrm{WW}$ & 756 & 5 & 0.007 & $1.93(1.32-2.81)$ & A E G P \\
\hline
\end{tabular}

$A$ age, $B X$ needle biopsy, $C I$ confidence interval, $C O N T$ continuous, $E$ extent, $G$ Gleason grade, $n s$ not significant, $P$ prostate-specific antigen, $P C S$ prostate cancer-specific survival, $P C R$ prostate cancer recurrence, $P x$ pathological features, $R P$ radical prostatectomy, $R X$ radiotherapy, $T$ clinical stage, $T x$ treatment, TURP transurethral resection of the prostate, $W W$ watchful waiting

To our knowledge, our multicentre study is the largest to assess the prognostic impact of Ki-67 in a cohort of diagnostic biopsies. In our previous cohort including conservatively managed patients, we demonstrated the discriminatory value of Ki-67 on transurethral resection of the prostate and needle biopsies [5, 6]. However, this current cohort is larger, clinically more contemporary with Gleason grade reviewed according to last ISUP recommendations [21] and confirmed our first results. 
In this study, we performed Ki-67 on needle biopsies instead of radical prostatectomies or transurethral resection of the prostate specimens to approach conditions for clinical decision before any treatment. The assessment of Ki-67 on the whole slides rather than tissue micro arrays reflects routine use and avoids potential bias due to tumor heterogeneity. Indeed, studies comparing the assessment of Ki-67 on tissue micro arrays and on whole slides recommended the use of the latter $[22,23]$.

The Ki-67 cut-off varies from a study to another. In our series, we identified 5\% $\mathrm{Ki}-67$ as the most relevant cut-off given the results of our cut-point analysis (Receiver Operating Characteristics curve). This cut-off aligns with the most frequently used level, fixed around $5-10 \%[4,5,18,24]$ and the difference may be explained by the constitution of the cohort, the material or the method of assessment. In addition, Kaplan-Meier plots confirmed robust curve separation between low and high $\mathrm{Ki}-67$ groups at 5\% cut-off.

For the first time, we report a subgroup analysis of Ki-67 prognostic impact in grade groups. Ki-67 was the most discriminatory in grade groups 1 and 2 compared to higher grade groups (Forest Plot). These low-grade groups with the best prognosis are frequently included in active surveillance studies [25]. For these patients, Ki-67 expression could help discriminate the most aggressive from the majority of indolent cancers.

The weaknesses of our study include its retrospective nature and the use of cases dating from 1990 to 2003. Nevertheless, only long follow-up time enables appropriate assessment of cancer specific survival that was our endpoint. Metastasis free survival would have been a useful addition that is missing in our study. However, cancer specific survival is a major endpoint for any biomarker and is reproducible to compare studies. In needle biopsies cohort, the amount of cancer available in needle biopsies can be limited that could impact tumor representativeness and the assessment of multifocality. The immunohistochemistry itself has several limitations including the antibody, preanalytic and analytic steps lowered by its worldwide use in several other tumors and its reproducible interpretation [10].

Ki-67 was mostly assessed by one pathologist. However, the inter-observer concordance in Ki-67 assessment is good and could be further improved by the use of cut-offs [10]. In addition, visual scoring of $\mathrm{Ki}-67$ and corresponding $\mathrm{H} \& \mathrm{E}$ slide was used instead of digital scoring as inflammation and high grade PIN in prostate biopsies are frequent pitfalls. In particular, inflammatory cells are frequently stained by Ki-67 immunohistochemistry and included in digital analysis. In addition, the two different approaches were compared in prostate cancer with a high inter-correlation agreement [26].

In conclusion, Ki-67 immunoexpression independently predicted prostate cancer patient outcome, adjusted for standard clinico-pathological parameters, in a cohort of diagnostic biopsies. If incorporated into routine practice, this assessment might assist clinicians in discriminating the most aggressive prostate cancer, improving treatment selection especially in low-grade cancer. Nevertheless, owing to the limitations of this study, a prospective validation of our findings in independent series is required to confirm its clinical utility.

Acknowledgements We gratefully acknowledge the support from Cancer Research UK, ORCHID, a SPORE grant from the US National Cancer Institute (P50CA09629), the David H. Koch Fund and Myriad Genetics. We thank investigators and staff in the cancer registries and participating hospitals for their support.

\section{Compliance with ethical standards}

Conflict of interest There are no conflicts of interest to disclose.

Publisher's note: Springer Nature remains neutral with regard to jurisdictional claims in published maps and institutional affiliations.

\section{References}

1. Wilt TJ, Brawer MK, Jones KM, Barry MJ, Aronson WJ, Fox S, et al. Radical prostatectomy versus observation for localized prostate cancer. N Engl J Med. 2012;367:203-13.

2. Hamdy FC, Donovan JL, Lane JA, Mason M, Metcalfe C, Holding P, et al. 10-Year Outcomes after Monitoring, Surgery, or Radiotherapy for Localized Prostate Cancer. N Engl J Med. 2016;375:1415-24.

3. Wiegel T, Bartkowiak D, Bottke D, Bronner C, Steiner U, Siegmann A, et al. Adjuvant radiotherapy versus wait-and-see after radical prostatectomy: 10-year follow-up of the ARO 96-02/AUO AP 09/95 trial. Eur Urol. 2014;66:243-50.

4. Berlin A, Castro-Mesta JF, Rodriguez-Romo L, HernandezBarajas D, Gonzalez-Guerrero JF, Rodriguez-Fernandez IA, et al. Prognostic role of $\mathrm{Ki}-67$ score in localized prostate cancer: A systematic review and meta-analysis. Urol Oncol. 2017; 35:499-506.

5. Berney DM, Gopalan A, Kudahetti S, Fisher G, Ambroisine L, Foster CS, et al. Ki-67 and outcome in clinically localised prostate cancer: analysis of conservatively treated prostate cancer patients from the Trans-Atlantic Prostate Group study. Br J Cancer. 2009;100:888-93.

6. Fisher G, Yang ZH, Kudahetti S, Moller H, Scardino P, Cuzick J, et al. Prognostic value of $\mathrm{Ki}-67$ for prostate cancer death in a conservatively managed cohort. $\mathrm{Br} \mathrm{J}$ Cancer. 2013;108: 271-7.

7. Epstein JI. An update of the Gleason grading system. J Urol. 2010;183:433-40.

8. Epstein JI, Zelefsky MJ, Sjoberg DD, Nelson JB, Egevad L, Magi-Galluzzi C, et al. A Contemporary Prostate Cancer Grading System: A Validated Alternative to the Gleason Score. Eur Urol. 2016;69:428-35.

9. Dowsett M, Nielsen TO, A'Hern R, Bartlett J, Coombes $\mathrm{RC}$, Cuzick J, et al. Assessment of $\mathrm{Ki} 67$ in breast cancer: recommendations from the International Ki67 in Breast Cancer working group. J Natl Cancer Inst. 2011;103: 1656-64.

10. Raap M, Liessem S, Ruschoff J, Fisseler-Eckhoff A, Reiner A, Dirnhofer S, et al. Quality assurance trials for Ki67 assessment in pathology. Virchows Arch: Int J Pathol. 2017;471:501-8. 
11. Hoogland AM, Kweldam CF, van Leenders GJ. Prognostic histopathological and molecular markers on prostate cancer needlebiopsies: a review. Biomed Res Int. 2014;2014:341324.

12. Cuzick J, Swanson GP, Fisher G, Brothman AR, Berney DM, Reid JE, et al. Prognostic value of an RNA expression signature derived from cell cycle proliferation genes in patients with prostate cancer: a retrospective study. Lancet Oncol. 2011;12:245-55.

13. Stattin P, Damber JE, Karlberg L, Bergh A. Cell proliferation assessed by Ki-67 immunoreactivity on formalin fixed tissues is a predictive factor for survival in prostate cancer. $\mathrm{J}$ Urol. 1997; 157:219-22.

14. Li R, Heydon K, Hammond ME, Grignon DJ, Roach M, 3rd, Wolkov HB, et al. Ki-67 staining index predicts distant metastasis and survival in locally advanced prostate cancer treated with radiotherapy: an analysis of patients in radiation therapy oncology group protocol 86-10. Clin Cancer Res. 2004; 10:4118-24.

15. Pollack A, DeSilvio M, Khor LY, Li R, Al-Saleem TI, Hammond ME, et al. Ki-67 staining is a strong predictor of distant metastasis and mortality for men with prostate cancer treated with radiotherapy plus androgen deprivation: Radiation Therapy Oncology Group Trial 92-02. J Clin Oncol. 2004;22:2133-40.

16. Khor LY, Bae K, Paulus R, Al-Saleem T, Hammond ME, Grignon DJ, et al. MDM2 and Ki-67 predict for distant metastasis and mortality in men treated with radiotherapy and androgen deprivation for prostate cancer: RTOG 92-02. J Clin Oncol. 2009;27:3177-84.

17. Tollefson MK, Karnes RJ, Kwon ED, Lohse CM, Rangel LJ, Mynderse LA, et al. Prostate cancer Ki-67 (MIB-1) expression, perineural invasion, and gleason score as biopsy-based predictors of prostate cancer mortality: the Mayo model. Mayo Clin Proc. 2014;89:308-18.

18. Tretiakova MS, Wei W, Boyer HD, Newcomb LF, Hawley S, Auman H, et al. Prognostic value of Ki67 in localized prostate carcinoma: a multi-institutional study of $>1000$ prostatectomies. Prostate Cancer Prostatic Dis. 2016;19:264-70.
19. Lobo J, Rodrigues A, Antunes L, Graca I, Ramalho-Carvalho J, Vieira FQ, et al. High immunoexpression of Ki67, EZH2, and SMYD3 in diagnostic prostate biopsies independently predicts outcome in patients with prostate cancer. Urol Oncol. 2018; 36:161 e7-e17.

20. Green WJ, Ball G, Hulman G, Johnson C, Van Schalwyk G, Ratan HL, et al. KI67 and DLX2 predict increased risk of metastasis formation in prostate cancer-a targeted molecular approach. Br J Cancer. 2016;115:236-42.

21. Epstein JI, Egevad L, Amin MB, Delahunt B, Srigley JR, Humphrey PA, et al. The 2014 International Society of Urological Pathology (ISUP) Consensus Conference on Gleason Grading of Prostatic Carcinoma: Definition of Grading Patterns and Proposal for a New Grading System. Am J Surg Pathol. 2016;40: 244-52.

22. Tramm T, Kyndi M, Sorensen FB, Overgaard J, Alsner J. Influence of intra-tumoral heterogeneity on the evaluation of BCL2, Ecadherin, EGFR, EMMPRIN, and Ki-67 expression in tissue microarrays from breast cancer. Acta Oncol. 2018;57:102-6.

23. Yang Z, Tang LH, Klimstra DS. Effect of tumor heterogeneity on the assessment of Ki67 labeling index in well-differentiated neuroendocrine tumors metastatic to the liver: implications for prognostic stratification. Am J Surg Pathol. 2011;35: 853-60.

24. Leon P, Cancel-Tassin G, Drouin S, Audouin M, Varinot J, Comperat $\mathrm{E}$, et al. Comparison of cell cycle progression score with two immunohistochemical markers (PTEN and Ki-67) for predicting outcome in prostate cancer after radical prostatectomy. World J Urol. 2018.

25. Briganti A, Fossati N, Catto JWF, Cornford P, Montorsi F, Mottet N, et al. Active Surveillance for Low-risk Prostate Cancer: The European Association of Urology Position in 2018. Eur Urol. 2018;74:357-68

26. Richardsen E, Andersen S, Al-Saad S, Rakaee M, Nordby Y, Pedersen MI, et al. Evaluation of the proliferation marker Ki-67 in a large prostatectomy cohort. PLoS ONE 2017;12:e0186852. 